\section{Régis Guillaume (dir.) Globalisation, systèmes productifs et dynamiques territoriales : regards croisés au Québec et dans le Sud-Ouest français, Paris L'harmattan, 2005,327 p.}

Q uelle belle coïncidence que de retrouver dans un quatrième chapitre de cet ouvrage collectif une contribution de deux auteures, qui, à leur façon, sur la base de leurs propres observations et à partir de leurs références bibliographiques, en arrivent sensiblement à la même démonstration de la (re)construction territoriale effectuée par PierreAndré Julien dans l'ouvrage présenté plus haut. Cette contribution, aussi intéressante par l'effort de conceptualisation réalisé que par la nature des informations issues d'un travail de terrain, à mon avis, aurait dû se retrouver au tout début du volume. Une affirmation formulée sans porter préjudice à la qualité de la contribution des auteurs québécois responsables du premier chapitre portant sur les interrelations universitéindustrie à Montréal dans la reconversion à l'économie du savoir. J'y devine un geste de courtoisie de la part de R. Guillaume, maître de conférences au département de géographie de Toulouse-Le Mitrail et chercheur au Centre interdisciplinaire d'études urbaines. En plus de sa propre contribution, le responsable de cet effort collectif a bien su coordonner le travail de pas moins de vingt-deux auteurs dont neuf Québécois aux noms familiers des lecteurs d'O\&T : J.-L. Klein, D.G. Tremblay, J.-M. Fontan., C. Manzagol, D. Doloreux, C. Bryant, etc.

Avant d'ouvrir ce volume, je pensais bien faire en portant essentiellement mon attention aux contributions des auteurs de chez nous sous le prétexte que si le Sud-Ouest de la mère patrie ne manque pas de charmes, ce qui s'y passe se trouve loin de nos préoccupation. Erreur. Ne fusse que sur le plan conceptuel, on y trouve d'intéressantes considérations, en introduction, comme cette définition d'un territoire : un processus de construction permanente susceptible de se voir éclairer à partir de dimensions historiques, sociales, politiques et bien sûr économiques et dont les logiques s'expriment à des échelles plurielles. On y trouve des relations entre entreprises en marche qui induisent nécessairement une proximité géographique. Cette dernière, favorise la coordination « d'agents situés » qui mettent en œuvre des stratégies de nature différente. On l'aura compris, selon R. Guil- laume, l'objectif de l'ouvrage vise : « [...] à relever et décrypter, dans un contexte de tension exacerbée et d'affaiblissement des outils de régulation traditionnels, l'expression de nouveaux enjeux ». Il est bien précisé que les sites étudiés sont analysés à partir d'une grille qui tient compte de leurs caractéristiques spécifiques et des initiatives locales qui s'expriment, en France comme au Québec, dans le cadre d'une accélération des processus de décentralisation.

Le quatrième chapitre, où Pierre-andré Julien va se reconnaître, s'intitule: "Réseaux d'acteurs locaux pour des projets de territoire : le cas des secteurs électrique et métallurgique pyrénéens ». À la suite d'une définition de ce que sont les systèmes productifs locaux, les deux maîtres (on ne dit pas maîtresses...) de conférences de Tarbes s'intéressent aux modes d'organisation de la production au niveau territorial. Ceci, en reconnaissant que de nos jours l'offre de ressources représente un potentiel émanant d'acteurs locaux à travers des processus d'apprentissage et d'innovation. En se plaçant dans le cadre du (re-) développement des territoires, les deux auteures son conduites à analyser les rapports dialectiques tissés entre les acteurs et leurs espaces d'insertion. Cette démarche les amène à s'interroger sur les territoires qui se construisent dans l'articulation d'acteurs locaux, privés et publics, et sur quelles ressources territoriales ils s'appuient pour asseoir leur développement. La démonstration d'un territoire en reconstruction se fait ici à partir de deux organisations résiliaires: le secteur électromagnétique d'Adour et un centre de recherche en électronique de puissance. On est loin, comme on le voit, de la problématique de nos chères régions ressources, où le 2 par 4 est encore bien présent... Notons leur conclusion : la valorisation d'un territoire dépend de trois facteurs, à savoir le temps, les réseaux et la spécificité. S’y trouve cité un auteur que Pierre-André Julien pourrait ajouter à sa liste.

Ceci étant dit, je doute que mon collègue ajoute à ses références bibliographiques le chapitre se rapportant à la Beauce dû à Doloreux, Filion et Klein, car, de toute évidence, ces auteurs ne semblent pas le moins du monde vouloir se faire les promoteurs de la création de nouvelles Beauce. À leurs yeux, le tissu institutionnel et les réseaux d'échange d'informations de cette région mythique sont peu développés. S’ils reconnaissent la présence évidente d'une multiplication de PME autour d'un entrepreneuriat local et familial et l'existence d'un fort sentiment d'appartenance, l'en- 
vers de la médaille prend différentes formes : faibles salaires, sous-scolarisation de la main-d'œuvre, dépendance envers les donneurs d'ordres et les centres extérieurs. Ils y voient un système de production local dont la régulation n'est pas économique (absence d'intégration verticale, de partenariat de valeur ajoutée, de réseau innovateur (p. 225). Pour eux, la région de la Beauce, si elle peut se voir considérer comme innovatrice, elle ne correspond pas au modèle classique du milieu innovateur (cf. les travaux du GREMI). Y domine un secteur manufacturier traditionnel à faible valeur ajoutée, souvent spécialisé dans la transformation de ressources naturelles. Les innovations observées sont de type incrémental plutôt que de type radical. Une enquête conduite par Doloreux révèle que, dans $90 \%$ des cas, l'innovation concerne l'amélioration secondaire d'un procédé déjà existant. Les auteurs dégagent que la Beauce ne peut-être identifiée comme un système d'innovation technologique. Faut donc aller voir ailleurs.

Et cet ailleurs, ce peut être le fameux Triangle d'or de la Montérégie étudié par D.G. Tremblay en portant attention aux stratégies de diversification. Cet intéressant chapitre traite du rôle de la proximité dans les relations interentreprises. À l'instar de ce que démontrent plusieurs études effectuées au sein de l'INRPME (Institut de recherche sur les PME) de l'UQTR, l'auteure signale que les entreprises, en majorité, considèrent que leur principal avantage concurrentiel réside du côté de la différenciation des produits et de la qualité, beaucoup moins du côté des prix.

Enfin, notons que le lecteur trouvera sur le secteur du lait au Québec, et celui du foie gras, non pas du lac Brome mais du sud-ouest de l'Hexagone, tout ce qu'il désirait savoir sans oser le demander. Oui, un livre étonnant pour un ouvrage collectif faisant appel à une gamme aussi variée d'auteurs. N'ayant rien à voir avec un vin de garde, il est à consommer maintenant, mais il sera encore bon dans deux ou trois ans.

André Joyal

Université du Québec à Trois-Rivières 
Notes 
Notes 